Article

\title{
Performance Improvement of Dual-Pulse Heterodyne Distributed Acoustic Sensor for Sound Detection
}

\author{
Xiangge He ${ }^{1,2} \oplus$, Min Zhang ${ }^{2,3}$, Lijuan Gu ${ }^{1,2, *} \mathbb{C}$, Shangran Xie ${ }^{4}$, Fei Liu ${ }^{2}$ and Hailong Lu ${ }^{1,2}$ \\ 1 College of Engineering, Peking University, Beijing 100871, China; hexiangge@pku.edu.cn (X.H.); \\ hlu@pku.edu.cn (H.L.) \\ 2 Beijing International Center for Gas Hydrate, Peking University, Beijing 100871, China; \\ zhang_min@pku.edu.cn (M.Z.); liufei19900213@126.com (F.L.) \\ 3 Institute of Ocean Research, Peking University, Beijing 100871, China \\ 4 Max Planck Institute for the Science of Light, Staudtstr. 2, 91058 Erlangen, Germany; \\ shangran.xie@mpl.mpg.de \\ * Correspondence: glj0317@pku.edu.cn; Tel.: +86-1591-023-6616
}

Received: 28 December 2019; Accepted: 9 February 2020; Published: 13 February 2020

\begin{abstract}
Phase fading is fatal to the performance of distributed acoustic sensors (DASs) influencing its capability of distributed measurement as well as its noise level. Here, we report the experimental observation of a strong negative correlation between the relative power spectrum density (PSD) at the heterodyne frequency and the noise floor of the detected phase for the heterodyne demodulated distributed acoustic sensor (HD-DAS) system. We further propose a weighted-channel stack algorithm (WCSA) to alleviate the phase fading noise via an enhancement of the relative PSD at the heterodyne frequency. Experimental results show that the phase noise of the demodulated signal can be suppressed by $13.7 \mathrm{~dB}$ under optimal condition. As a potential application, we exploited the improved HD-DAS system to retrieve a piece of music lasted for $205 \mathrm{~s}$, demonstrating the reliability of detecting wideband sound signal without distortion.
\end{abstract}

Keywords: distributed acoustic sensor; Rayleigh backscattering; phase fading; sound detection

\section{Introduction}

Acting as the sensing element, optical fiber has several inherent advantages including immunity to electromagnetic radiation, high flexibility, low cost and so on. As a result, distributed optical fiber sensors, with the optical fiber functioning as both sensing and transmitting medium, have attracted great attention from scientific and industrial communities [1,2]. Since distributed optical fiber sensor is based on light scattering originated from fluctuations in the physical properties of the sensing fiber, it can be categorized into three groups based on Raman scattering [1], Brillouin scattering [3], and Rayleigh scattering [4], respectively. Among all those approaches, distributed acoustic sensor (DAS) exploiting coherent Rayleigh backscattering to acquire the acoustic-wave-induced strain variation on the fiber has been extensively explored over the last decade $[5,6]$.

Since DAS can obtain the dynamic acoustic field along the sensing fiber with length of several kilometers, it is ideal for applications like structural health monitoring [7,8], intrusion detection [9], leak detection [10], geological sensing [11,12], and oilfield exploration [13]. Especially when applied in the oilfield, with harsh environment of high temperature and pressure, one can adopt the pre-installed optical fiber or optimize the optical fiber to meet special requirements. In this scenario, field tests of DAS applied in vertical seismic profile, micro-seismic monitoring, hydraulic fracturing monitoring, and reservoir surveillance have been reported [14,15]. Jousset et. al. recently used the DAS system to interrogate the pre-installed communication optical fiber cables to record seismic signals from 
natural and man-made sources on Reykjanes Peninsula, SW-Iceland, shedding a light on earth hazard assessment and exploration [16].

The detected signal of DAS comes from coherent interference between Rayleigh backscattered light from different positions along the fiber. Since the amplitude and the phase shift of the backscattered signal are randomly distributed over distance, ripple like fluctuations appear on top of the interference intensity. When the backscattered signal superimposes destructively, the detected signal is vanishingly small, which is known as the intensity fading. The fading effect is fatal in the DAS system because, at the fading positions, the acoustic signal is lost. To overcome this issue, Hartog et al. used multiple optical interrogation frequencies with diverse fading properties to aggregate the data obtained to substantially reduce the fading noise [17]. However, this scheme greatly increases the system complexity, especially at the source part. Chen et al. used an intensity modulator in their time-gated digital optical frequency domain reflectometry configuration based DAS system. The harmonics induced by the intensity modulator were fully adopted to suppress fading noise via using matched filter and rotated-vector-sum method [18].

Different from the previously mentioned single pulse configurations, we recently proposed a dual-pulse heterodyne demodulated DAS (HD-DAS) approach that can retrieve the acoustic-induced phase modulation in a distributed manner [19]. In this approach, a pulse pair with fixed frequency difference functions as a moving interferometer along the sensing fiber, and the phase information can be demodulated with a heterodyne detection algorithm. An extremely low noise floor and a high signal-to-noise ratio (SNR) can be achieved in this system due to the fact that the pulse pair share the noise properties when propagating along the same fiber. The phase fading effect was also characterized in the HD-DAS system and was found to be dominated by the random phase retardant rather than the scattering coefficient [20].

Here, in this work, we experimentally investigate the phase fading effect and propose a new approach on improving the HD-DAS system by alleviating the phase fading effect. Through a detailed signal analysis of the collected signal, it is found that, along the sensing fiber, the relative power spectrum density (PSD) at heterodyne frequency has a negative correlation relationship with the noise floor of the detected phase. In addition, the root mean square (RMS) of the phase noise increases sharply when the relative PSD at heterodyne frequency is lower than $50 \mathrm{~dB}$. Based on this observation, we proposed a weighted-channel stack algorithm (WCSA) to suppress the noise floor through the enhancement of the relative PSD at heterodyne frequency. Finally, we use the improved HD-DAS system to recover the acoustic signal induced by a piece of music demonstrating the effectiveness of the proposed approach.

\section{Working Principle of the HD-DAS System}

Figure 1 illustrates the working principle of HD-DAS system, in which a heterodyne pulse pair, offset in both temporal and frequency domains, are injected into the sensing optical fiber. The heterodyne pulse pair functions similar to the sensing and reference arms of an interferometer. Thus, the phase change induced by the external acoustic field is modulated onto the heterodyne frequency, which can be retrieved using a heterodyne demodulation algorithm [19]. As the two pulses propagate along the same fiber, the noise properties are shared with each other and thus self-cancelled. Therefore, this system can retrieve the demodulated signal with high signal-to-noise ratio (SNR) after heterodyne detection.

To numerically simulate the DAS system, the discrete model of Rayleigh backscattering is commonly adopted [21]. In this model, the optical fiber is considered as a series of discrete reflectors with a length much longer than the optical wavelength. The reflectivity of each reflector follows the Rayleigh distribution and the reflected phase is uniformly distributed in $[-\pi, \pi]$. The electric field of the backscattered pulse pair from position $z$ can be expressed as [20] 


$$
\begin{aligned}
E(z, \tau) & =\left(E_{1}+\delta E_{1}\right) \int_{z}^{z+\frac{w}{2}} r(p, \tau) e^{j \theta(p, \tau)} \exp \left[j 2 \int_{0}^{p} \phi(l, \tau) d l\right] e^{-j \omega_{1} \tau+j \delta \phi_{1}} d p \\
& +\left(E_{2}+\delta E_{2}\right) \int_{z+\frac{L_{d}}{2}}^{z+\frac{L_{d}+w}{2}} r(p, \tau) e^{j \theta(p, \tau)} \exp \left[j 2 \int_{0}^{p} \phi(l, \tau) d l\right] e^{-j \omega_{2} \tau+j \delta \phi_{2}} d p
\end{aligned}
$$

where $\tau=1 / f_{r}$ is the sampling time within each analog to digital converter (ADC) channel, and $f_{r}$ is repetition rate of the pulse pair. $E_{i}(i=1,2)$ is the field amplitudes of each pulse in the pulse pair with amplitude fluctuation of $\delta E_{i}$. The pulse pair are modulated to frequency of $f_{1}$ and $f_{2}$ with an identical pulse width of $w$. The frequency offset, i.e., the heterodyne frequency is given by $\Delta f=f_{1}-f_{2}$, while the spatial distance between them is $L_{d} \cdot \omega_{i}=2 \pi f_{i}(i=1,2)$ indicates their angular frequency. $r(p, \tau)$ and $\theta(p, \tau)$ are the reflectivity and phase of the reflector. $\phi(l, \tau)$ is the acoustic-induced phase variation at position $l$ and time $\tau$. The phase noise generated with pulse pair propagation are represented by $\delta \phi_{1}$ and $\delta \phi_{2}$. As shown in Equation (1), the detected signal has a complicated expression resulting from the interference between backscattered lights from different reflectors along the fiber. Since the scattering amplitude and phase shift of those Rayleigh scatters are randomly distributed along the sensing fiber, the collected interference signal appears as intensity fluctuations, which is known as fading effect.

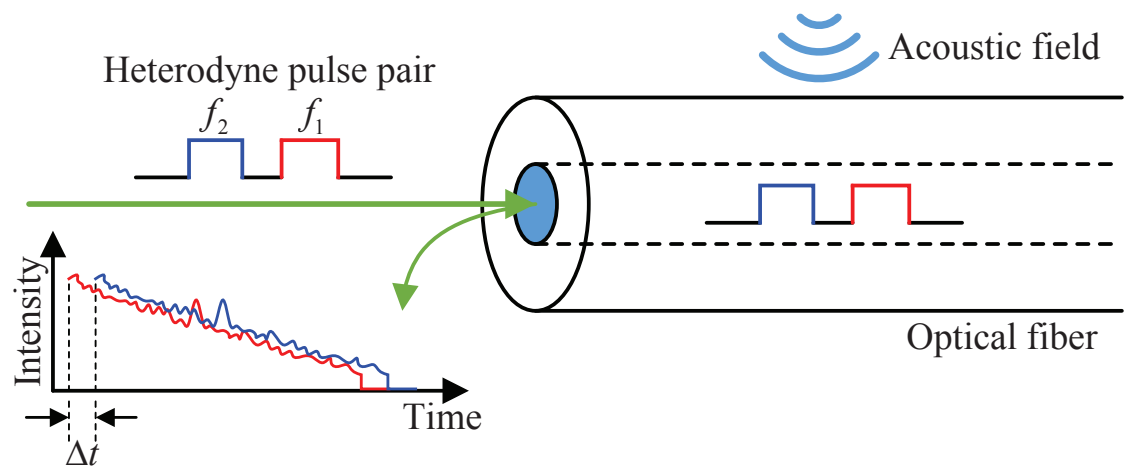

Figure 1. Working principle of the heterodyne demodulated distributed acoustic sensor (HD-DAS) system.

Figure 2 shows the configuration of our HD-DAS system. A narrow linewidth, approximately $100 \mathrm{~Hz}, \mathrm{CW}$ laser at central wavelength of $1550.12 \mathrm{~nm}$ (NKT Koheras BasiK E15, Denmark) is used as the light source. The narrow linewidth laser was used to suppress the laser phase noise. The output of the laser is divided into two optical paths by an optical coupler (OC1). Then, two acousto-optic modulators, AOM1 with frequency of $100 \mathrm{MHz}$ and AOM2 with frequency of $100.05 \mathrm{MHz}$, are used to generate the heterodyne pulses with a pulse width of $w=6 \mathrm{~m}$ and a repetition rate of $f_{r}=200 \mathrm{kHz}$. The heterodyne frequency is $\Delta f=50 \mathrm{kHz}$. A $L_{d}=10 \mathrm{~m}$ delay fiber is placed after AOM2 to temporally offset the two pulses. The heterodyne pulse pair is amplified by an Erbium-doped fiber amplifier (EDFA1) before injecting into the sensing fiber through the optical fiber circulator. The Rayleigh backscattered signal is boosted by another EDFA before sending it into the photo detector (PD). Then, a high speed data acquisition card (DAQ) samples the data with a rate of $100 \mathrm{MS} / \mathrm{s}$. Finally, the data are transmitted to a computer to implement the heterodyne demodulation algorithm [19]. 


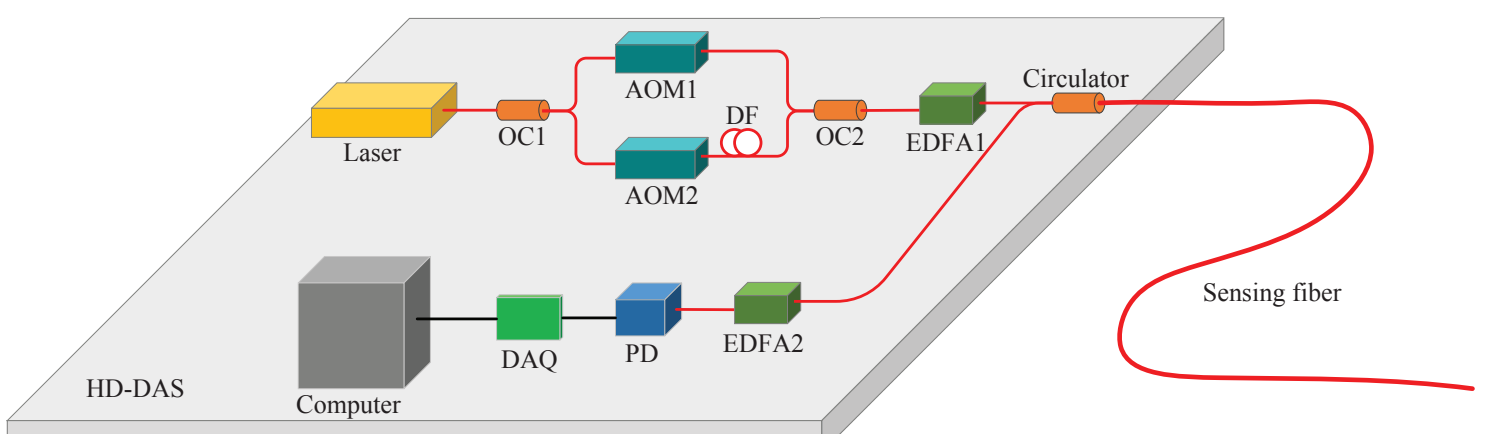

Figure 2. Experimental configuration of HD-DAS system. OC, optical coupler; AOM, acousto-optic modulator; DF, delay fiber; EDFA, Erbium-doped fiber amplifier; PD, photo detector; DAQ, data acquisition card.

\section{Results and Discussions}

\subsection{The Phase Fading Phenomenon in the HD-DAS System}

The $500 \mathrm{~m}$ sensing fiber is isolated in a box and the waterfall plot of the demodulated phase signal under steady condition is shown in Figure 3b, along with the root mean square (RMS) value of the retrieved phase at different positions plotting in Figure 3a. The standard deviation of the RMS is $\sim 0.72$ rad. We notice that the retrieved phase signal fluctuates randomly along the sensing fiber and also varies with time. Note that the common mode noises in the system (including laser intensity and phase noises, AOM modulation noise and environmental noise) partially contribute to the phase noise, while they are not the key reason for the phase fading since they are identical to all sampling channels. The inhomogeneity of the phase noise mainly results from the phase fading noise [20] inherent to the DAS system. In addition, the phase fading noises also vary with time indicating that the parameter of $r$ and $\theta$ in Equation (1) are functions of time. This further causes the position of the fading channels fluctuates over time.

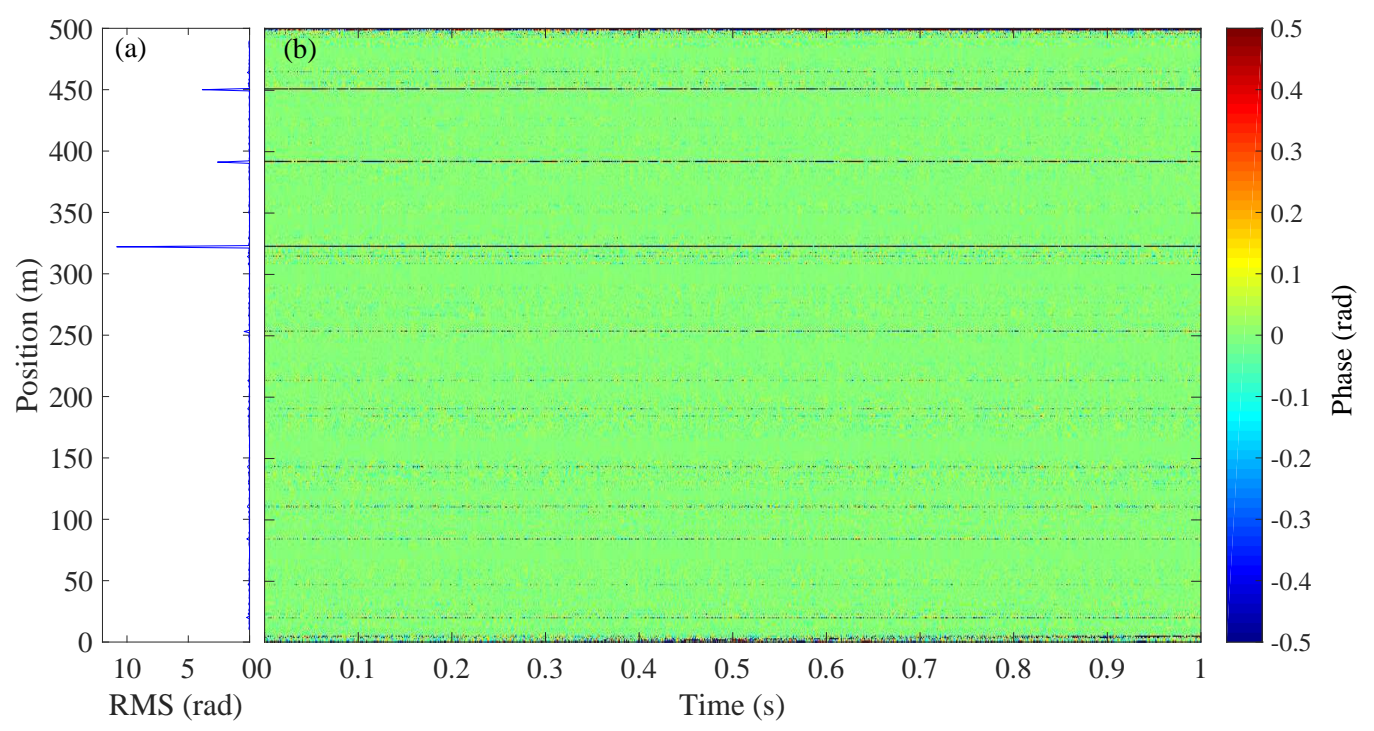

Figure 3. (a) RMS and (b) waterfall plot of the demodulated phase signal over position.

Using a $100 \mathrm{MS} / \mathrm{s}$ ADC sampling frequency, the sampling length for each channel is $1 \mathrm{~m}$ and the entire $500 \mathrm{~m}$ long optical fiber contains 500 sampling channels. Two typical channels (channel 163 with low noise and channel 314 with high noise) with distinct noise levels are therefore chosen to be analyzed and compared. 
Figure 4 summarizes the results from channel 163, in which Figure 4a is the collected intensity signal and Figure $4 \mathrm{~b}$ is its PSD. The $1 \mathrm{~s}$ time duration is chosen as a compromise between negligible time-dependent effect of RMS and the sufficient frequency resolution to resolve the PSD value at the heterodyne frequency. The red dashed line in Figure $4 \mathrm{~b}$ marks the noise floor (estimated as the averaged PSD value from $1 \mathrm{kHz}$ to $49 \mathrm{kHz}$ ) and the red diamond marks the PSD value at the heterodyne frequency $\Delta f$, i.e., $50 \mathrm{kHz}$. The resultant relative PSD at the heterodyne frequency is $\triangle P S D_{\Delta f}=80.4 \mathrm{~dB}$ defined as the difference between the PSD at $\Delta f\left(-12.2 \mathrm{~dB} \mathrm{~V}^{2} / \mathrm{Hz}\right)$ and the noise floor $\left(-92.6 \mathrm{~dB} \mathrm{~V}^{2} / \mathrm{Hz}\right)$. Figure $4 \mathrm{c}$ plots the demodulated phase signal along with its PSD shown in Figure $4 \mathrm{~d}$. The corresponding noise floor, calculated as the averaged PSD value from $100 \mathrm{~Hz}$ to $20 \mathrm{kHz}$, is $-83.5 \mathrm{~dB} \mathrm{rad} / 2 \mathrm{~Hz}$. The visible peak at $10 \mathrm{kHz}$ (as well as the harmonics in Figure $4 \mathrm{~b}$ ) is caused by the unavoidable switching effect in the AOM pulse modulator.
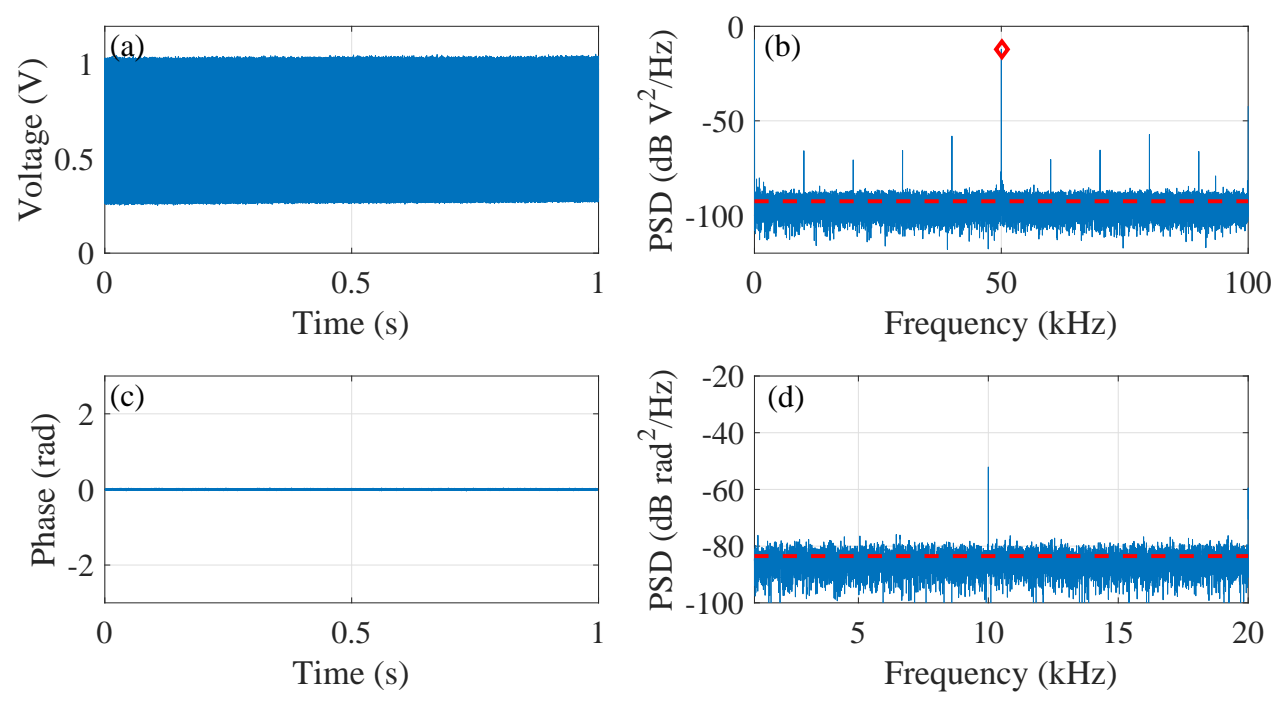

Figure 4. Results for channel 163 (low noise channel). (a) the detected intensity signal; (b) the PSD of the intensity signal; (c) the demodulated phase signal; (d) the PSD of the phase signal.

As a comparison, Figure 5 plots results for channel 314. In this case, the noise floor of the intensity signal is $-98.8 \mathrm{~dB} \mathrm{~V}^{2} / \mathrm{Hz}$ and the PSD at $\Delta f$ is $-47.0 \mathrm{~dB} \mathrm{~V}^{2} / \mathrm{Hz}$, giving $\triangle P S D_{\Delta f}=51.8 \mathrm{~dB}$. The noise floor of the demodulated phase in this case is $-54.6 \mathrm{~dB} \mathrm{rad} / 2 \mathrm{~Hz}$, which is much higher than that in channel 163.

\subsection{Relation between PSD and Noise Floor}

The blue line in Figure 6a plots the noise floor of the demodulated phase signal over position. The same dataset as shown in Figure 3 was used. Meanwhile, the relative PSD at the heterodyne frequency is also plotted in the same figure (red line). It is clear that the relative PSD at heterodyne frequency is anti-correlated with the noise floor of the detected phase. The estimated cross-correlation coefficient is $\sim-0.99$. 

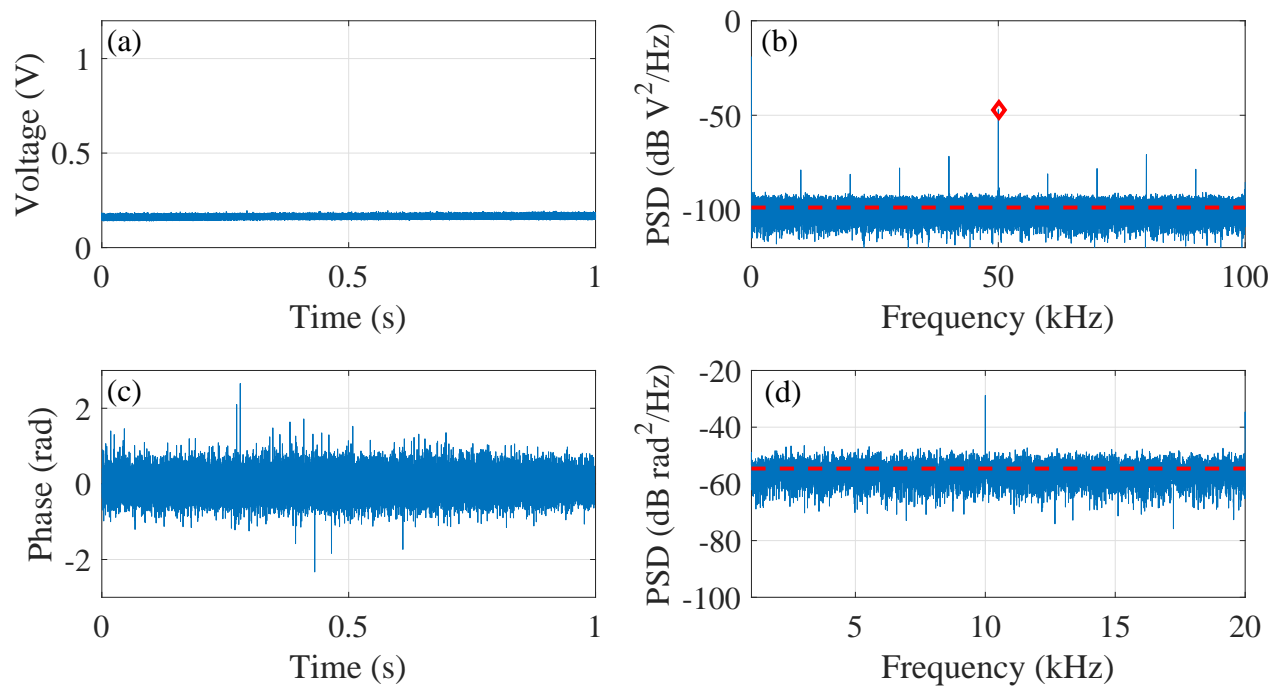

Figure 5. Results for channel 314 (high noise channel). (a) the detected intensity signal; (b) the PSD of the intensity signal; (c) the demodulated phase signal; (d) the PSD of the phase signal.

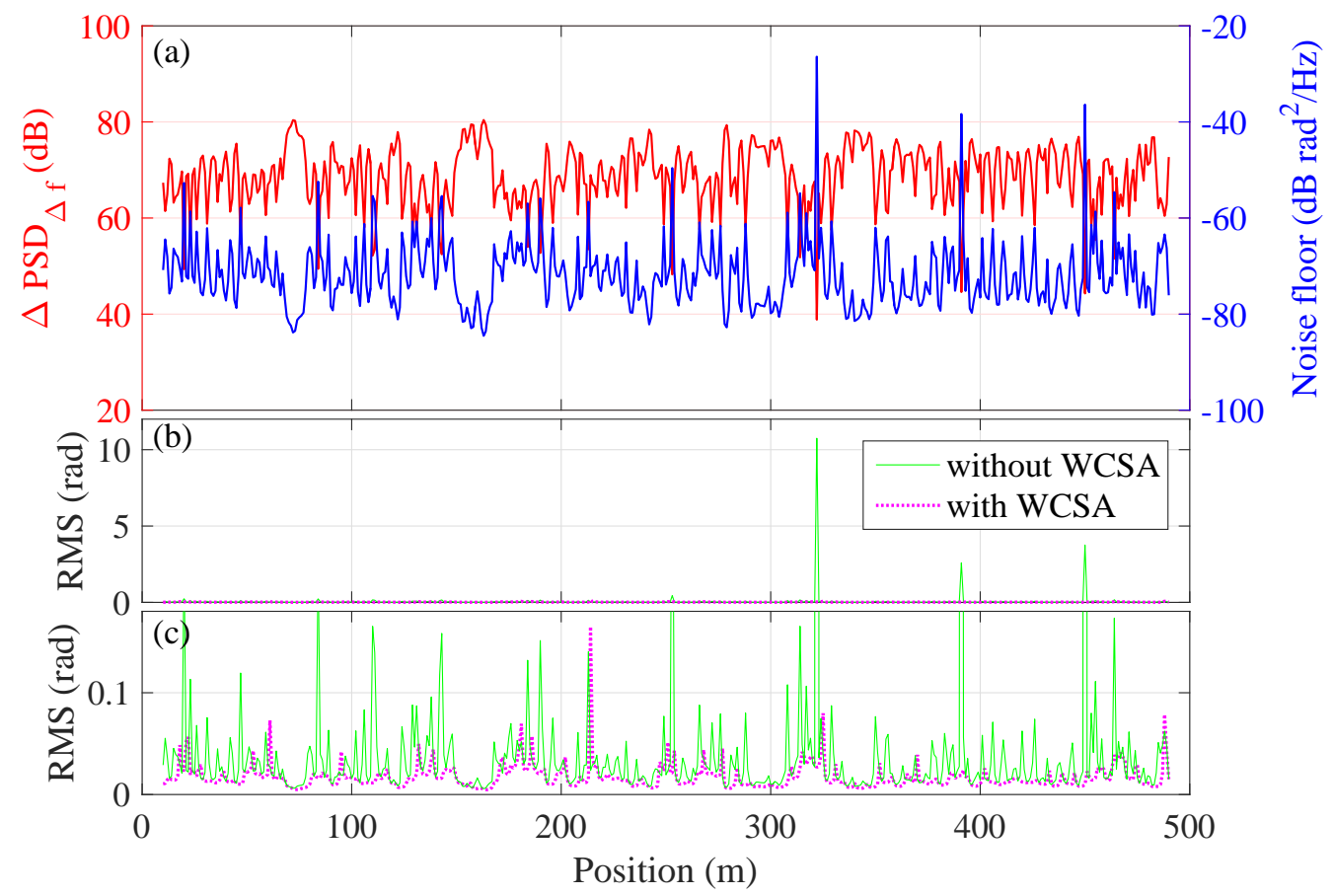

Figure 6. (a) the relative PSD at heterodyne frequency (left vertical axis) and the corresponding noise floor of the detected phase (right vertical axis) at different positions, showing a clear anti-correlation relation; (b) the RMS value of the detected phase over position without (green-solid) and with (magenta-dashed) WCSA; (c) zoom-in of (b) in vertical axis.

To provide more insight on the observation, the blue curve in Figure 7 plots the signal-to-noise ratio (SNR) of the collected intensity signal at different positions. Here, SNR is estimated as:

$$
S N R=\frac{P_{s}}{P_{n}}=\frac{\int_{f_{s 1}}^{f_{s 2}} P S D}{\int_{f_{n 1}}^{f_{n 2}} P S D}
$$


where $P_{s}$ and $P_{n}$ are the power level of signal and noise, respectively, PSD represents the power spectrum density of the intensity signal. $\left[f_{s 1}, f_{s 2}\right]$ and $\left[f_{n 1}, f_{n 2}\right]$ are the considered frequency range of signal and noise, respectively. In the calculation, the bandwidth of signal is $[49.9 \mathrm{kHz}, 50.1 \mathrm{kHz}]$ with the rest considered as noise. As can be seen from Figure 7, the relative PSD at the heterodyne frequency is strongly linked with the SNR (with the estimated cross-correlation coefficient of $\sim 0.98$ ). This is reasonable since the relative PSD at heterodyne frequency is the difference between the PSD at $\Delta f$ and the noise floor. In Gabai's work [22], it has been found that the SNR of the demodulated phase was directly proportional to the SNR of the backscattered trace. According to Equation (2), the higher the noise floor, the lower the SNR of the demodulated phase, explaining the negative relation between the relative PSD at heterodyne frequency and the noise floor.

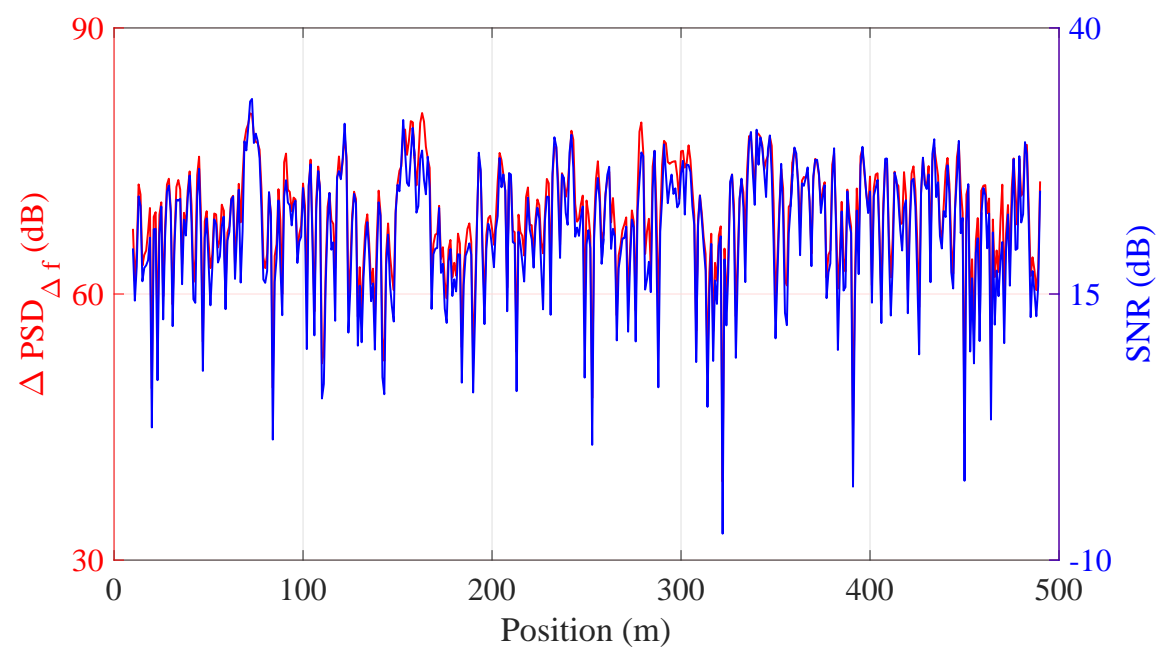

Figure 7. Measured relative PSD at heterodyne frequency (left vertical axis) and the SNR (right vertical axis) over position.

Figure $6 \mathrm{c}$ shows the RMS value, i.e., the effective amplitude, of the detected phase change over position. It can be seen that, in certain channels, the RMS increases sharply due to the phase fading noises. Figure 8 displays the RMS of the detected phase versus the relative PSD at heterodyne frequency. These data were calculated from a 10-s continuously measured signal. It can be seen that the RMS value increases sharply when the relative PSD at heterodyne frequency is lower than $50 \mathrm{~dB}$ (red dashed line).

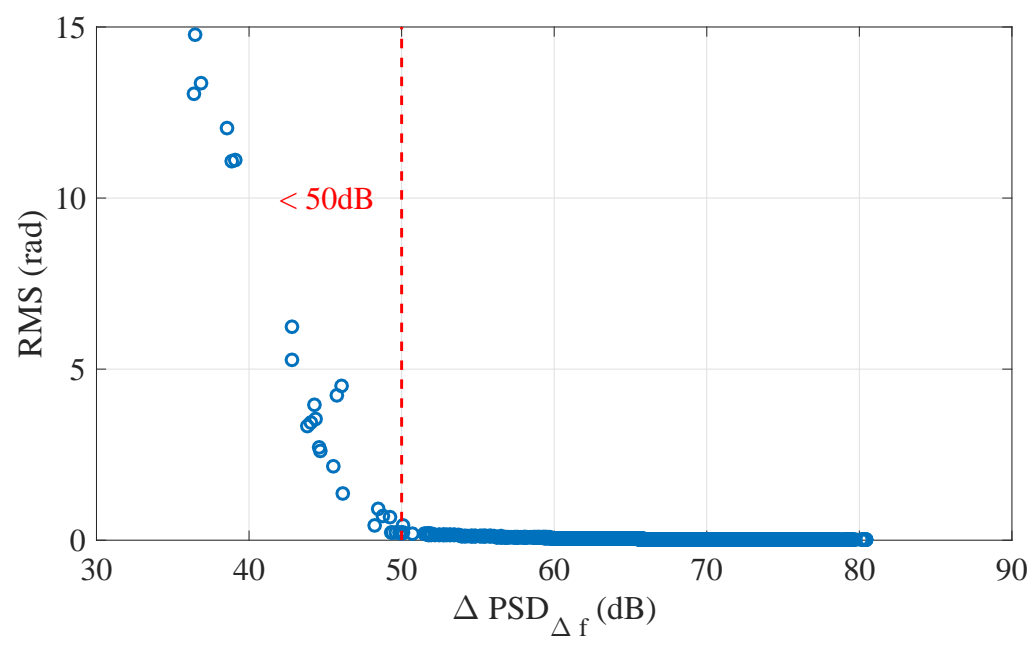

Figure 8. The RMS of the detected phase versus the relative PSD at heterodyne frequency. 


\subsection{Weighted-Channel Stack Algorithm}

Based on the above observations, we propose a weighted-channel stack algorithm (WCSA) to alleviate the phase fading effect in the HD-DAS system. The procedure of the algorithm is listed as follows:

- Convert the intensity data collected by DAQ $I(t)$ into parallel data $I(z, \tau)$, where $t$ and $\tau$ refer to the fast-time and slow-time frame [20]. The position $z$ corresponds to a specific sampling channel.

- Divide $I(z, \tau)$ into blocks with time interval of $0.1 \mathrm{~s}$ for further processing. Then, we have $I(z, \tau)=\left\{I\left(z, \tau_{n}\right) \mid \tau_{n}=((n-1) * 0.1 s, n * 0.1 s], n=1,2, \cdots\right\}$. Note that we used the graphics processing unit (GPU) of the computer for parallel processing of the demodulation algorithm, in which case a $0.1 \mathrm{~s}$ time interval was found to be optimal for data processing.

- Calculate the PSD of $I(z, \tau)$ at different channels of each time block; furthermore, find the PSD of heterodyne frequency $\operatorname{PSD}_{\Delta f}^{n}\left[I\left(z, \tau_{n}\right)\right]$ and its noise floor $N F^{n}\left[I\left(z, \tau_{n}\right)\right]$ within each time block. Calculate the relative PSD at heterodyne frequency of different channels using Equation (3):

$$
\Delta P S D_{\Delta f}^{n}(z)=P S D_{\Delta f}^{n}\left[I\left(z, \tau_{n}\right)\right]-N F^{n}\left[I\left(z, \tau_{n}\right)\right]
$$

- Define the weight of different channels using Equation (4):

$$
w^{n}(z)= \begin{cases}\Delta P S D_{\Delta f}^{n}(z), & \Delta P S D_{\Delta f}^{n}(z) \geq 50 d B \\ 0, & \Delta P S D_{\Delta f}^{n}(z)<50 d B\end{cases}
$$

- The weighted-channel stacked signal can be obtained by Equation (5), where $M$ is the number of channels used for stacking. Note that a larger value of $M$ may reduce the spatial resolution of the sensor due to the average effect:

$$
I\left(z, \tau_{n}\right)=\sum_{k=z-M}^{z} w^{n}(k) I\left(k, \tau_{n}\right)
$$

- With the heterodyne demodulation algorithm proposed in [19], the demodulated phase signal can be retrieved.

We then follow the aforementioned procedure to re-demodulate the signal in Figure 3 . The result is given in Figure 9. Here, the number of channels for stacking is $M=4$, which is the optimal stacking number for our data set. Figure 9a shows the RMS of the phase signal over position and the standard deviation of the RMS is $0.15 \mathrm{rad}$, achieving a noise reduction of $13.7 \mathrm{~dB}$ compared to the results in Figure 3 (also see Figure $6 b$,c for a direct comparison over position). Note that the increase of noise in some channels (e.g., $220 \mathrm{~m}$ ) may be induced by the noise in adjacent channels after applying WSCA; the overall effect of noise suppression, however, is clear.

\subsection{Sound Detection}

\subsubsection{Experimental Setup}

As an application, we used our improved HD-DAS system for sound detection. Figure 10a,b illustrate respectively the schematic and physical diagrams of the experimental configuration. At a position of $50 \mathrm{~m}$, a segment of $5 \mathrm{~m}$ fiber was set close to a noise source. The piece of the fiber that used to 'listen' to the music was fixed to the clamps by adhesive tape. The distance between two clamps was approximately $30 \mathrm{~cm}$ and the fiber locates at position of about $92 \mathrm{~m}$. A music player was placed underneath the fiber to play the music. 


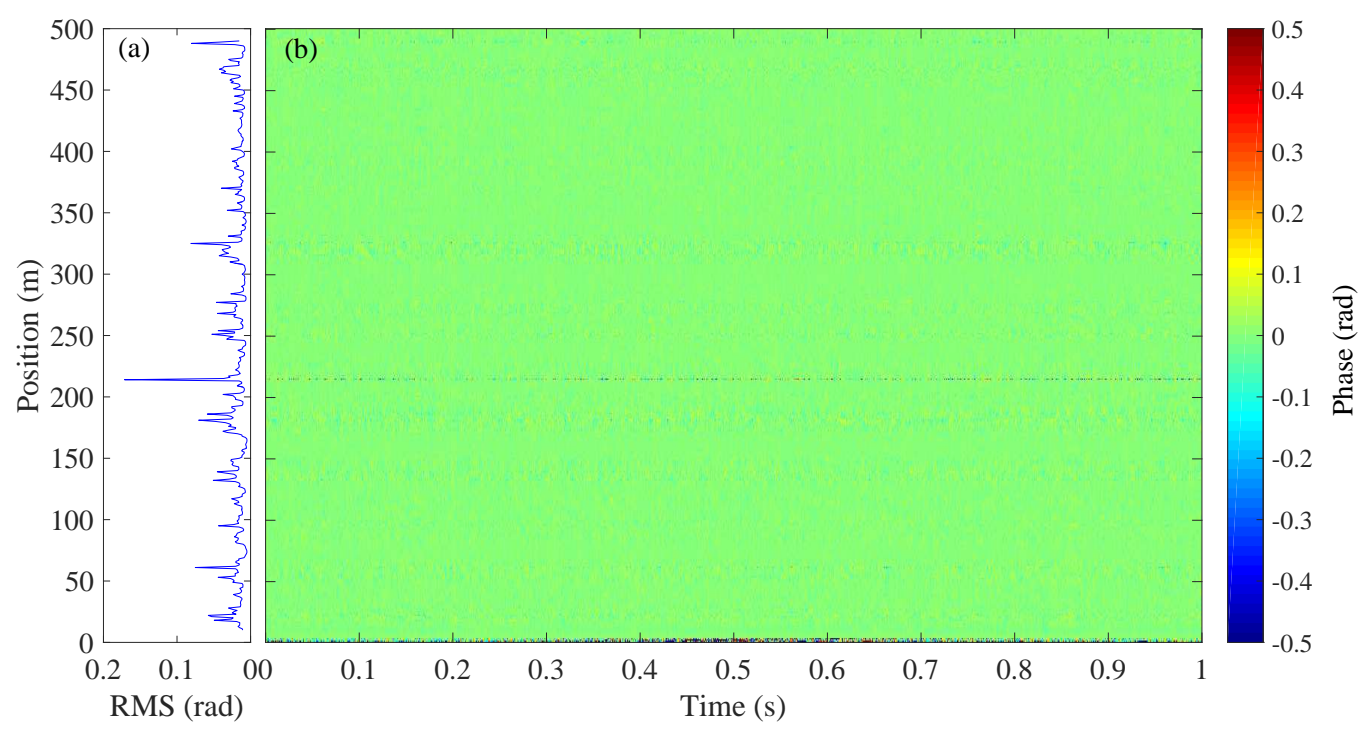

Figure 9. (a) RMS and (b) waterfall plot of the demodulated phase over position with WCSA applied.
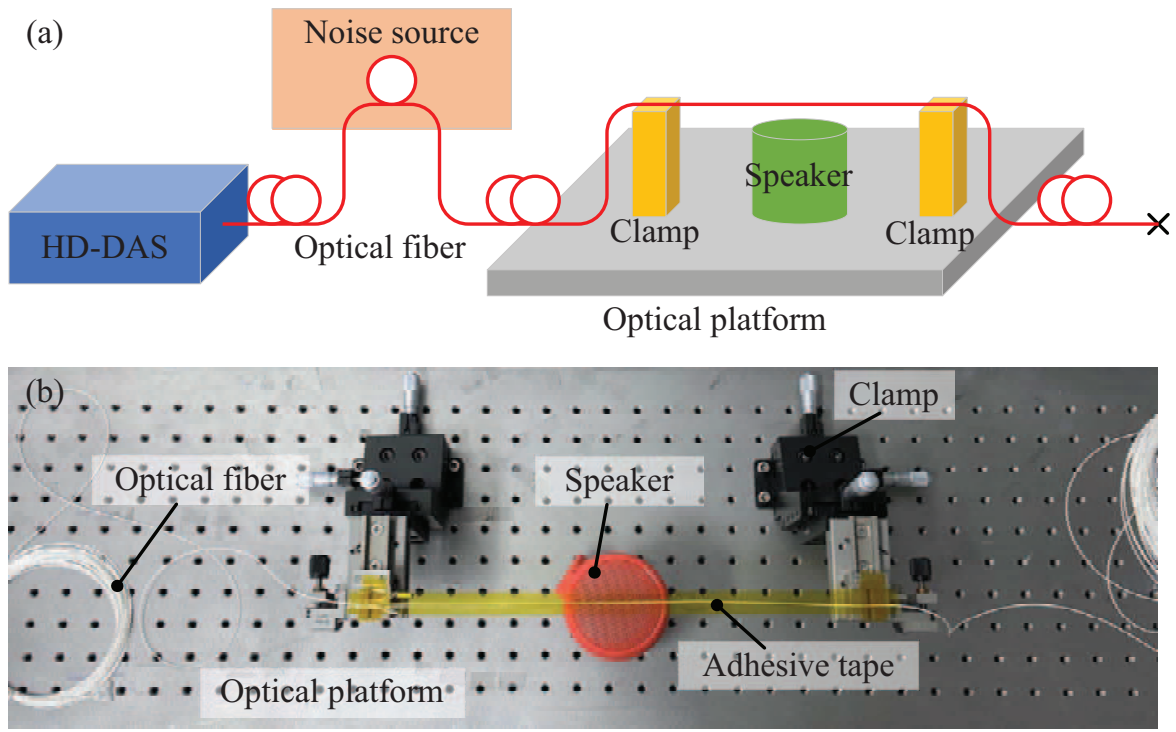

Figure 10. The experimental configuration using the HD-DAS system for sound detection. (a) schematic diagram; (b) real system in the lab.

\subsubsection{Sound Detection Results}

Figure 11 displays the waterfall plot of the retrieved phase signal from the improved HD-DAS system (thus with WCSA algorithm applied). Two sets of vibration signals are visible in the figure: one is located at position $50 \mathrm{~m}$, which is the noise-induced phase change; the other at $92 \mathrm{~m}$ represents the music-induced phase change. The gauge length can be calculated as $\left(w+L_{d}\right) / 2$, which is $8 \mathrm{~m}$ in this case. This is in accordance with the length of signal span in the waterfall plot. Note that the noise source is set far away from the music player and whose effect on the music-induced phase signal is negligible.

Figure 12 plots the retrieved noise signal located at position $50 \mathrm{~m}$. It can be seen that the noise source is mainly the $50 \mathrm{~Hz}$ electronic frequency and its harmonics. The noise at high frequency can be considered as white noise. 


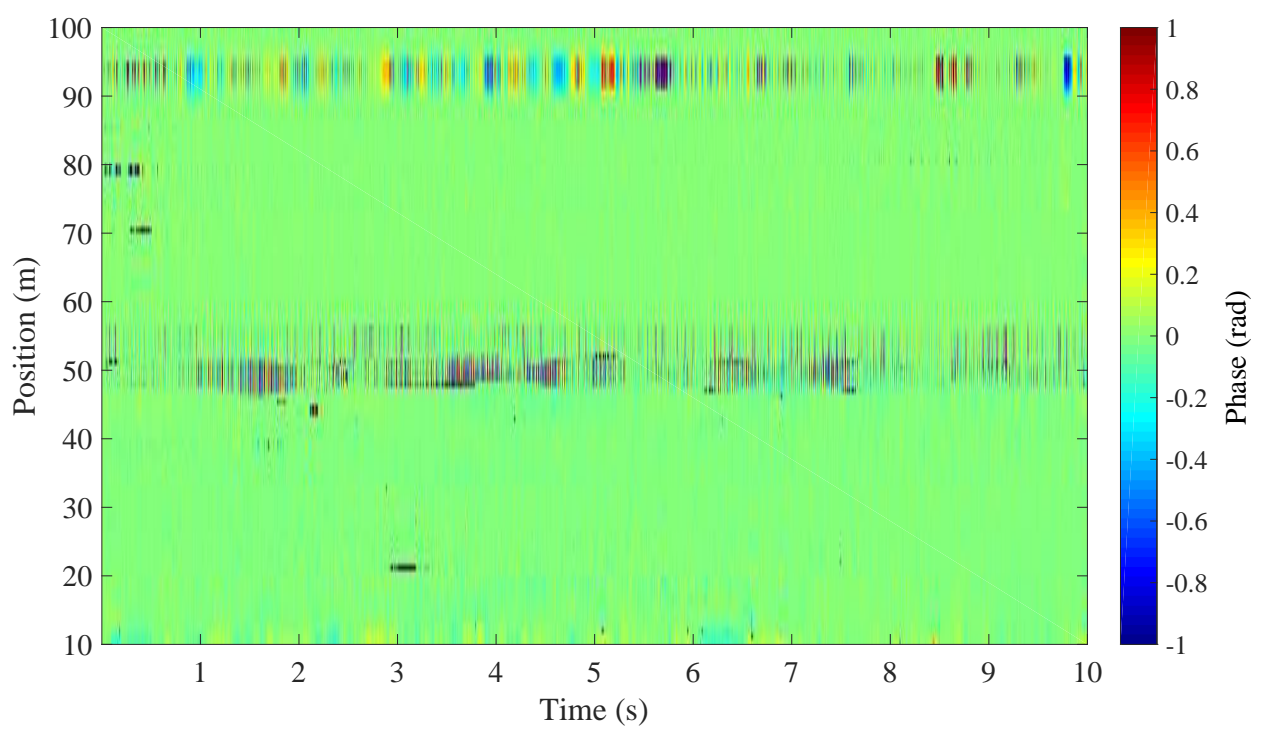

Figure 11. Waterfall plot of the detected phase signal for the music.
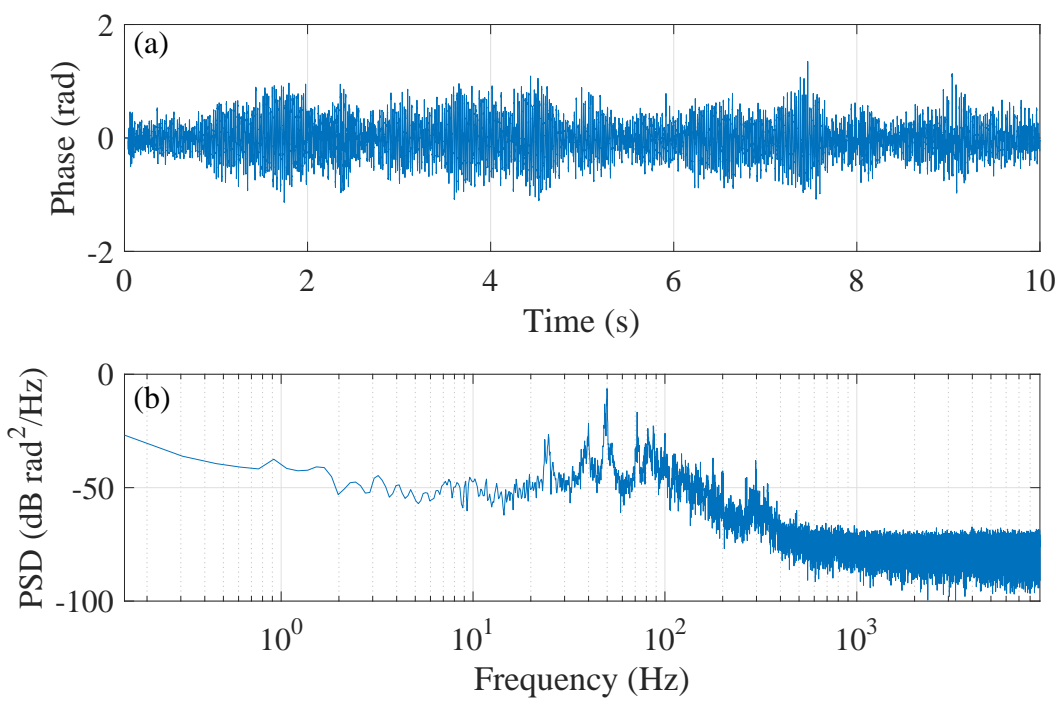

Figure 12. Plot of (a) the demodulated phase signal located at position $50 \mathrm{~m}$ and (b) its PSD.

Figure 13 shows the retrieved music signals lasted for $205 \mathrm{~s}$ with and without WCSA. When WCSA is not used, the phase fading effect exists that can be clearly identified from the demodulated signal at $85 \mathrm{~s}$ and $177 \mathrm{~s}$, where the music signals are submerged in the sharply increased phase noises. The retrieved music without WCSA can refer to the Supplementary retrievedSound1.wav and the noise interruption can be identified from the audio. The retrieved sound with WCSA is also provided in Supplementary retrievedSound2.wav and, in this case, smooth and beautiful music without distortion is obtained. As described previously, the phase fading noise will change with time, while, with the proposed algorithm, the $205 \mathrm{~s}$ music signal can be retrieved continuously without distortion, validating the fading alleviation effect of this algorithm.

As a direct comparison, in Figure 14a, we plot the retrieved sound signal from time $85 \mathrm{~s}$ to $87 \mathrm{~s}$ without (red) and with (blue) WCSA applied, overlapped with the original waveform (black). As can be seen, without WCSA, the retrieved signal shows fatal distortions due to a phase fading effect. Under this circumstance, the PSD of the retrieved phase (shown in Figure 14b) appears significant deviation from that of the original data (especially at low frequency range). On the other hand, when WCSA is used, the PSD of the retrieved phase coincides very well with that of the applied sound. 

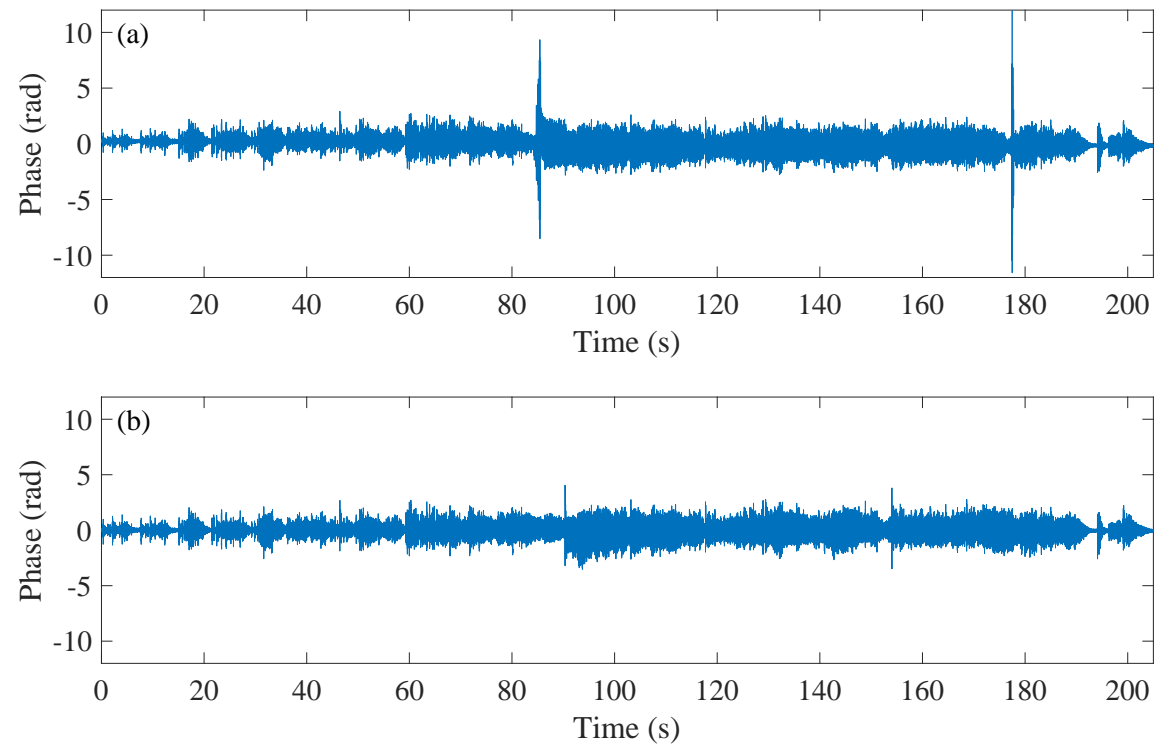

Figure 13. The plot of the retrieved sound signals. (a) the retrieved phase signal without WCSA; (b) the retrieved phase signal with WCSA.
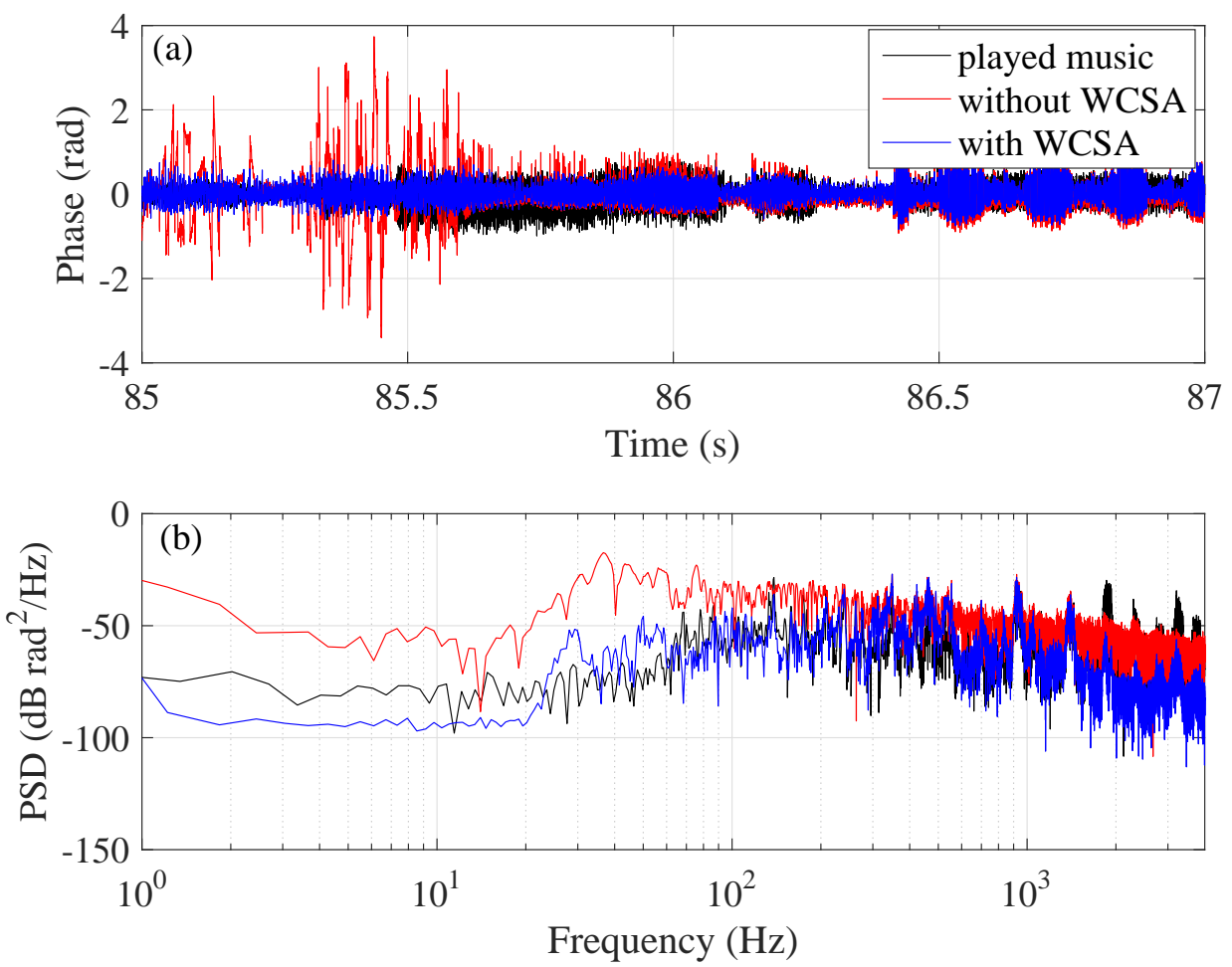

Figure 14. Comparison of the retrieved sound signals without (red) and with (blue) WCSA applied. The black curve is the original music data. (a) time-domain signals; (b) the PSD of the signals.

\section{Conclusions}

In conclusion, we report the experimental observation of an anti-correlation relation between the relative PSD at heterodyne frequency and the noise floor of the detected phase signal in the HD-DAS system. A novel WCSA algorithm was further proposed to suppress the phase fading noise, with the key to enhance the relative PSD at heterodyne frequency. An optimal reduction of noise level of 
$13.7 \mathrm{~dB}$ can be achieved using our approach. We have also demonstrated the improved performance of the system on sound detection. The improved HD-DAS system offers a promising solution to seismic wave detection in down-hole and oceanic environments.

Supplementary Materials: The following are available online at http:/ /www.mdpi.com/1424-8220/20/4/999/s1, Sound S1: retrievedSound1.wav, Sound S2: retrievedSound2.wav.

Author Contributions: Conceptualization, X.H.; writing-original draft preparation, X.H.; writing-review and editing, L.G., S.X., and F.L.; supervision, M.Z. and H.L. All authors have read and agreed to the published version of the manuscript.

Funding: This research was funded by the financial support from China Geological Survey with project No. DD20190234.

Conflicts of Interest: The authors declare no conflict of interest.

\section{Abbreviations}

The following abbreviations are used in this manuscript:

DAS Distributed acoustic sensor

HD-DAS Heterodyne demodulated distributed acoustic sensor

SNR signal-to-noise ratio

PSD Power spectrum density

RMS Root mean square

WCSA Weighted-channel stack algorithm

\section{References}

1. Bao, X.; Chen, L. Recent Progress in Distributed Fiber Optic Sensors. Sensors 2012, 12, 8601-8639. doi:10.3390/s120708601. [CrossRef] [PubMed]

2. Lu, P.; Lalam, N.; Badar, M.; Liu, B.; Chorpening, B.T.; Buric, M.P.; Ohodnicki, P.R. Distributed optical fiber sensing: Review and perspective. Appl. Phys. Rev. 2019, 6, 041302. doi:10.1063/1.5113955. [CrossRef]

3. Ukil, A.; Braendle, H.; Krippner, P. Distributed Temperature Sensing: Review of Technology and Applications. IEEE Sens. J. 2012, 12, 885-892. doi:10.1109/JSEN.2011.2162060. [CrossRef]

4. Peled, Y.; Motil, A.; Tur, M. Fast Brillouin optical time domain analysis for dynamic sensing. Opt. Express 2012, 20, 8584-8591. doi:10.1364/OE.20.008584. [CrossRef]

5. Lu, Y.; Zhu, T.; Chen, L.; Bao, X. Distributed Vibration Sensor Based on Coherent Detection of Phase-OTDR. J. Lightwave Technol. 2010, 28, 3243-3249. doi:10.1109/JLT.2010.2078798. [CrossRef]

6. Bao, X.; Zhou, D.P.; Baker, C.; Chen, L. Recent Development in the Distributed Fiber Optic Acoustic and Ultrasonic Detection. J. Lightwave Technol. 2017, 35, 3256-3267. [CrossRef]

7. Barrias, A.; Casas, J.R.; Villalba, S. A Review of Distributed Optical Fiber Sensors for Civil Engineering Applications. Sensors 2016, 16, 748. doi:10.3390/s16050748. [CrossRef]

8. Chen, Z.; Zhou, X.; Wang, X.; Dong, L.; Qian, Y. Deployment of a Smart Structural Health Monitoring System for Long-Span Arch Bridges: A Review and a Case Study. Sensors 2017, 17, 2151. doi:10.3390/s17092151. [CrossRef]

9. Gao, J.; Jiang, Z.; Zhao, Y.; Zhu, L.; Zhao, G. Full distributed fiber optical sensor for intrusion detection in application to buried pipelines. Chin. Opt. Lett. 2005, 3, 633-635.

10. Chelliah, P.; Murgesan, K.; Samvel, S.; Chelamchala, B.R.; Tammana, J.; Nagarajan, M.; Raj, B. Looped back fiber mode for reduction of false alarm in leak detection using distributed optical fiber sensor. Appl. Opt. 2010, 49, 3869-3874. doi:10.1364/AO.49.003869. [CrossRef]

11. Lindsey, N.J.; Martin, E.R.; Dreger, D.S.; Freifeld, B.; Cole, S.; James, S.R.; Biondi, B.L.; Ajo-Franklin, J.B. Fiber-Optic Network Observations of Earthquake Wavefields. Geophys. Res. Lett. 2017, 44, 11,792-11,799, doi:10.1002/2017GL075722. [CrossRef]

12. Daley, T.; Miller, D.; Dodds, K.; Cook, P.; Freifeld, B. Field testing of modular borehole monitoring with simultaneous distributed acoustic sensing and geophone vertical seismic profiles at Citronelle, Alabama. Geophys. Prospect. 2016, 64, 1318-1334, doi:10.1111/1365-2478.12324. [CrossRef] 
13. He, X.; Pan, Y.; You, H.; Lu, Z.; Gu, L.; Liu, F.; Yi, D.; Zhang, M. Fibre optic seismic sensor for down-well monitoring in the oil industry. Measurement 2018, 123, 145-149. doi:10.1016/j.measurement.2018.03.047. [CrossRef]

14. Mateeva, A.; Lopez, J.; Potters, H.; Mestayer, J.; Cox, B.; Kiyashchenko, D.; Wills, P.; Grandi, S.; Hornman, K.; Kuvshinov, B.; et al. Distributed acoustic sensing for reservoir monitoring with vertical seismic profiling. Geophys. Prospect. 2014, 62, 679-692, doi:10.1111/1365-2478.12116. [CrossRef]

15. Li, M.; Wang, H.; Tao, G. Current and Future Applications of Distributed Acoustic Sensing as a NewReservoir Geophysics Tool. Open Pet. Eng. J. 2015, 8, 272-281. [CrossRef]

16. Jousset, P.; Reinsch, T.; Ryberg, T.; Blanck, H.; Clarke, A.M.; Aghayev, R.; Hersir, G.P.; Henninges, J.; Weber, M.; Krawczyk, C.M. Dynamic strain determination using fibre-optic cables allows imaging of seismological and structural features. Nat. Commun. 2018, 9, 2509. [CrossRef]

17. Hartog, A.; Liokumovich, L.; Ushakov, N.; Kotov, O.; Dean, T.; Cuny, T.; Constantinou, A.; Englich, F. The use of multi-frequency acquisition to significantly improve the quality of fibre-optic-distributed vibration sensing. Geophys. Prospect. 2018, 66, 192-202. [CrossRef]

18. Chen, D.; Liu, Q.; He, Z. High-fidelity distributed fiber-optic acoustic sensor with fading noise suppressed and sub-meter spatial resolution. Opt. Express 2018, 26, 16138-16146. [CrossRef]

19. He, X.; Xie, S.; Liu, F.; Cao, S.; Gu, L.; Zheng, X.; Zhang, M. Multi-event waveform-retrieved distributed optical fiber acoustic sensor using dual-pulse heterodyne phase-sensitive OTDR. Opt. Lett. 2017, 42, 442-445. doi:10.1364/OL.42.000442. [CrossRef]

20. He, X.; Zhang, M.; Xie, S.; Gu, L.; Liu, F.; Chen, Z.; Tao, Q. Identification and observation of the phase fading effect in phase-sensitive OTDR. OSA Contin. 2018, 1, 963-970. doi:10.1364/OSAC.1.000963. [CrossRef]

21. Park, J.; Lee, W.; Taylor, H.F. Fiber optic intrusion sensor with the configuration of an optical time-domain reflectometer using coherent interference of Rayleigh backscattering. Proc. SPIE 1998, 3555, 49-56.

22. Gabai, H.; Eyal, A. On the sensitivity of distributed acoustic sensing. Opt. Lett. 2016, 41, 5648-5651. [CrossRef] [PubMed]

(C) 2020 by the authors. Licensee MDPI, Basel, Switzerland. This article is an open access article distributed under the terms and conditions of the Creative Commons Attribution (CC BY) license (http://creativecommons.org/licenses/by/4.0/). 